\title{
Competencias profesionales del docente universitario desde una perspectiva integral
}

\section{Professional Skills of University Teachers from an Integral Perspective}

\author{
Marllelis Gutiérrez Hinestroza ${ }^{1}$, Marianela Silva Sánchez ${ }^{1}$, Sadi Iturralde Kure ${ }^{1}$ y María Caridad Mederos \\ Machado ${ }^{1}$ \\ ${ }^{1}$ Universidad Estatal Península de Santa Elena \\ *mgutierrez@upse.edu.ec
}

DOI: https://doi.org/10.26871/killkana_social.v3i1.443

\begin{abstract}
Resumen
El estudio tuvo como objetivo generar perspectivas teóricas para fundamentar el perfil integral de competencias profesionales de los docentes de los Institutos Universitarios de Tecnología y elaborar un perfil de competencias profesionales para dichos docentes, según sus propias consideraciones. La investigación se enmarcó en un enfoque mixto (cuantitativo-cualitativo) comenzando con una descripción de los hechos, conceptualización con su correspondiente análisis interpretativo (hermenéutica). La población estuvo conformada por 144 sujetos aplicando la fórmula de Sierra y Bravo quedó una muestra de 75 sujetos. Se utilizaron dos instrumentos: un cuestionario y una entrevista. Para la validez se utilizó el juicio de expertos y discriminante mediante el método de Cronbach. Se utilizó el programa de análisis estadístico SPSS versión 10, la técnica ANOVA y la prueba Rangos de Tukey. Para la entrevista se realizó un análisis interpretativo aplicado a los directivos. Entre las conclusiones más relevantes se puede indicar los profesores le dan preeminencia al aspecto docente, se requiere la implementación de un proceso pedagógico integral que proyecte el desarrollo de las distintas dimensiones humanas y le brinde significación a la práctica pedagógica.
\end{abstract}

Palabras clave: Competencias profesionales, Docente universitario, Investigación, Extensión.

\begin{abstract}
The objective of the study was to generate theoretical perspectives as a basis for the integral profile of the professional competences of Technology universityand institutes teachers; and to elaborate a professional competences profile for these teachers, according to their own considerations. The research was framed in a mixed approach (quantitativequalitative) beginning with a description of the facts and the conceptualization with its corresponding interpretative analysis (hermeneutic). The population consisted of 144 individuals, after applying the Sierra and Bravo formula, the sample was reduced to 75 participants. Two instruments were used: a questionnaire and an interview. For validity, expert judgement and discrimination using the Cronbach method were used. Additionally, the statistical analysis program SPSS version 10, the technique ANOVA and Tukey's Ranges test were used. For the interview, an interpretative analysis applied to the directors was carried out. One of the most relevant conclusions is that the professors give more importance to the teaching aspect. The implementation of an integral pedagogical process is required in order to project the development of the different human dimensions, and provide meaning to the pedagogical practice.
\end{abstract}

Keywords: Professional skills, University teacher, Investigation, Extension.

\section{Introducción}

La formación de profesionales competentes y comprometidos con el desarrollo social constituye hoy en día una misión esencial de la Educación Superior. Cada día la sociedad demanda con más fuerza la formación de profesionales capaces no solo de resolver con eficiencia los problemas de la práctica profesional, sino también y fundamentalmente de lograr un desempeño profesional ético responsable.
La responsabilidad ciudadana y el compromiso social como valores asociados al desempeño profesional, y por tanto vinculados a la competencia profesional, constituyen el centro de atención en el proceso de formación que tiene lugar en las instituciones universitarias en la actualidad, la cual se expresa en la necesidad de transitar de una formación tecnocrática a una formación humanista.

Sin duda que la sociedad del conocimiento, en la que estamos insertos, está planteando diversas demandas 
en todos los ámbitos del quehacer humano. La Educación Superior, y en especial quienes desarrollan funciones docentes, no son ajenos a esto.

La problemática se plantea al descubrir que muchas Instituciones de Educación Superior están adoptando nuevos modelos o enfoques de formación, para lo cual rediseñan el currículo, mejoran su estructura organizacional, incorporan tecnologías a sus procesos formativos. Sin embargo, sus docentes son los mismos y sobre ellos no se hace mucho, en especial en los aspectos de formación.

Por esta razón, asalta la duda de si se está pensando seria y responsablemente en cuáles deben ser las competencias que el docente universitario debe tener para lograr un desenvolvimiento efectivo de su quehacer.

El éxito profesional depende de su actividad individual y el desempeño en las funciones que ejerce, las razones por las que un profesional triunfa tienen que ver con sus conocimientos y con su actitud, entusiasmo y niveles de motivación. De estas afirmaciones se deriva que los resultados de la vida personal y profesional de cada individuo son la consecuencia lógica de las conductas que asume frente a las diversas situaciones y del trabajo que emprenda diariamente.

Bajo esta premisa, se fundamentó el origen de esta investigación, que tiene por objetivo esencial elaborar un perfil de competencias profesionales del docente universitario de los Institutos Universitarios de Tecnología según las consideraciones de sus propios docentes.

\section{Marco teórico}

Con el propósito de fundamentar las perspectivas teóricas, así como plantear elementos conceptuales que faciliten visualizar mejor la realidad de los objetivos, sus elementos, alcances, categorías, se consideraron criterios de diversos autores relacionados con las competencias profesionales.

Los profesores universitarios tienen como peculiaridad que laboran en un alto centro de estudios, en el cual no se formaron como tal, es decir, que no se prepararon previamente como docentes, ya que no existe en el mundo ningún centro especializado en educar profesores para ser docentes en la universidad. En la mayoría de los casos, el claustro de profesores está integrado por profesionales egresados en la propia universidad o en otra similar y como resultado de sus ejecutorias como alumnos, se han quedado a ejercer la docencia o sus intereses personales y labores profesionales en la producción y/o los servicios los han acercado a los recintos universitarios.

Hay quienes se preguntan qué significa ser profesor universitario. Gewer, A. y Montero, L. (1996), cuya respuesta está relacionada con las características que lo identifican profesionalmente, lo cual está a su vez determinado por las condiciones históricas y los contextos institucionales en que desarrollan su labor. Es decir, que caracterizar al profesor universitario presupone tener en cuenta los momentos históricos actuales de inicios del siglo
XXI y las peculiaridades de las instituciones universitarias donde laboran, debido a que entre ellas existen diferencias estructurales y funcionales, en dependencia no solo de su nivel de desarrollo, historia y tradiciones, sino también de las áreas geográficas donde se encuentran insertadas, así como las condiciones sociales, políticas y culturales de los países a los cuales pertenecen. Y definen al profesor como todo aquel que enseña de una manera organizada, en virtud de la preparación académica adquirida, que acredita a través de algún título, en alguno de los niveles del sistema escolar o estudio reglado. Y proponen como características definitorias de la profesionalidad en la docencia las siguientes:

1) Delimitación de un ámbito específico de actuación.

2) Preparación técnica y científica para resolver los problemas propios de su ámbito de actuación.

3) Compromiso de actualización y perfeccionamiento de los conocimientos y habilidades que le son propios.

4) Unos ciertos derechos sociales como individuo y como colectivo profesional.

5) Autonomía de actuación.

6) Compromiso deontológico con la práctica docente.

El profesor universitario debe ser un educador, lo que significa trascender las funciones de ser un simple instructor, expositor o dictador de lecciones asequibles en los textos, por lo que necesita de conocimientos psicopedagógicos y conocer a sus alumnos. Rodríguez (1999).

El docente universitario debe ser competente desde una concepción humanista de la educación, lo que significa no solo ser un conocedor de la ciencia que explica sino también de los contenidos teóricos y metodológicos de la Psicología y la Pedagogía contemporáneas, que lo capacite para diseñar en sus disciplinas un proceso de enseñanzaaprendizaje potenciador del desarrollo de la personalidad del estudiante. González (2002).

Es decir que el profesor de la universidad debe ser un sujeto que orienta su actuación con independencia y creatividad sobre la base de una sólida motivación profesional, así como valores profesionales firmemente arraigados, es decir, con un profesionalismo ético. Cortina (2001).

Las competencias profesionales pueden ser definidas como aquellas cualidades de la personalidad que permiten la autorregulación de la conducta del sujeto a partir de la integración de los conocimientos científicos, las habilidades y las capacidades vinculadas con el ejercicio de su profesión, así como de los motivos, sentimientos, necesidades y valores asociados a ella, que permiten, faciliten y promueven un desempeño profesional eficaz y eficiente dentro de un contexto social determinado. Expresan un enfoque holístico de la personalidad en la unidad de lo cognitivo, afectivo y conductual. Ortiz (2002).

El término competencia constituye también un concepto integrador porque unifica en un solo término, los elementos ejecutivos e inductivos de la autorregulación de la personalidad dentro del proceso docente-educativo. 
Barrón (2000) divide las competencias de los profesores universitarios en docentes e investigativas. Sobre las primeras plantea varias dimensiones:

- Preparación para la docencia: la preparación curricular, actitudinal y una formación pedagógica esencial.

Una propuesta más integradora debe considerar a las competencias profesionales del docente universitario como expresión de las diferentes aristas y esferas de su actuación en el ámbito universitario y conformadas por subcompetencias o competencias específicas, como son:

- Competencia Didáctica: caracteriza la facilidad de trasladar de manera asequible (y no facilista) a los estudiantes los contenidos de las ciencias que imparte por difíciles que parezcan, sin distorsionarlos ni vulgarizarlos. No basta con el dominio de dichos contenidos, es esencial que el profesor sea capaz de "pedagogizarlos", pues la lógica de la ciencia no coincide siempre con la lógica de su impartición.

- Competencia Académica: expresa el dominio de las ciencias que aportan a su labor docente en las diferentes asignaturas y disciplinas que imparte, así como de la Psicología y la Pedagogía, como ciencias que explican y fundamentan su actuar cotidiano en la enseñanza y en la investigación acerca de su práctica educativa.

- Competencia Investigativa: el saber hacer investigativo despierta una actitud más crítica y reflexiva sobre su labor pedagógica, obliga a hacer coincidir la teoría con su práctica, incrementa sus sensibilidad para detectar aquellos problemas que requieren de investigaciones científicas para su solución, exige de una mejor planificación y organización de su trabajo y a un mejor uso de él, un incremento sustancial de su espíritu creativo y autocrítico, es más objetivo en sus juicios y más flexible para aceptar los cambios provocados por los procesos renovadores e innovadores, un mayor tiempo dedicado al estudio individual y a la lectura sobre temas de interés profesional y una reafirmación de sus motivaciones profesionales como docente y como investigador.

- Competencia Ética: refleja su autonomía moral como modelo a imitar por sus alumnos y colegas en cuanto a los valores profesionales que lo caracterizan como persona, como profesor universitario y a la vez como especialista en una rama del saber científico.

- Competencia Comunicativa: posee tres componentes: el uso eficiente de su lengua materna, tanto oral como por escrito; el dominio de una lengua extranjera, preferentemente el idioma inglés, al menos para extraer información de la literatura científica actualizada y el desarrollo de habilidades para ser un comunicador profesional por su oratoria, por la brillantez y exactitud de su discurso, así como por la calidad de sus escritos debido a la esencia educativa de su labor.

- Competencia Cultural: debe poseer una cultura general e integral en especial de carácter humanística, que le permita estar actualizado sobre los acontecimientos científicos, políticos, económicos, históricos, sociales, ideológicos, artísticos más acuciantes de su tiempo, tanto en el ámbito universal como regional y nacional.

- Competencia Tecnológica: el gran desarrollo de la ciencia y la tecnología contemporáneas, cuya manifestación más evidente y acelerada es el creciente proceso de informatización de la sociedad, debe reflejarse en el docente universitario en su explotación pertinente a través del uso de las nuevas tecnologías de la información y la comunicación, en función de su labor docente e investigativa como herramientas insustituibles.

Las competencias docentes no solo son las actividades que los profesores realizan cuando están en clase con sus alumnos. Estas suponen un conjunto de actividades que han de realizar para asegurar el aprendizaje de sus alumnos. Las características o rasgos de la enseñanza universitaria son:

- Presupone el dominio de un conjunto de conocimientos, métodos y técnicas científicas.

- Exige considerar la integración del proceso enseñanzaaprendizaje con la actividad de investigación.

- La evaluación debe ser integral permitiendo la participación del alumno.

- La referencia al alumnado es indispensable.

En efecto, el profesor universitario aprende su función docente mediante un proceso de socialización que es en parte: intuitivo, autodidacta y sigue la rutina específica. En referencia a la competencia profesional del docente, se puede decir que no se trata de los conocimientos, destrezas y actitudes que deben poseer los docentes para implementar su acción didáctica y lograr alcanzar un nivel competente como docente. Entre los distintos componentes esenciales para que un docente sea competente se requiere, según Tobón (2005):

\subsection{Conocimientos}

- Dominio del más alto nivel de su asignatura.

- Actualización de estos conocimientos y dominio de la metodología de la investigación

- Dominio de la pedagogía universitaria.

\subsection{Destrezas y Habilidades}

- Habilidades para la comunicación educativa

- Facilidad para la relación interpersonal.

- Destrezas docentes específicas: selección y secuenciación de contenidos a impartir, organización y estructura de los conocimientos, planificación a largo y corto plazo de las actividades docentes y de aprendizaje, selección de métodos didácticos.

\subsection{En cuanto a la formación pedagógica}

Por formación pedagógica se entiende no la simple capacidad para expresar los contenidos disciplinarios propios de una profesión, ni el simple manejo instrumental de métodos de enseñanza. Se entiende por formación pedagógica el 
desarrollo de competencias para la conceptualización, aplicación y experimentación de los conocimientos, métodos y estrategias para la enseñanza de los saberes específicos en contextos institucionales.

Esta formación corresponde al campo pedagógico, que se distingue del campo educativo como lo ha resaltado Zuluaga (1998); mientras lo educativo se enmarca en la producción y reproducción de la cultura y la ciencia en el ámbito de lo social, la pedagogía se refiere al saber pedagógico que da identidad al docente alrededor de las preguntas por los fines, los métodos, las estrategias didácticas, la selección de contenidos, las formas de evaluación, las metas, la relación con los alumnos con el saber, las instituciones y las prácticas.

Atendiendo a estas consideraciones, el papel del docente debe ser de ejecutor. Es necesario que intervenga directamente en prácticas investigativas, ya que se lo exigen los continuos cambios en la producción de conocimiento mediante procesos reflexivos para que su actividad profesional no quede reducida a un simple proceso de instrucción y transmisión de información con la finalidad de lograr las metas establecidas.

Esta dinámica ejecutoria debe orientarse a fomentar la investigación como práctica cotidiana en sus estudiantes, para facilitar un aprendizaje autónomo y crítico entre sus alumnos. Así esta experiencia ayudará a superar las dificultades y favorecerá encontrar respuestas que orienten el diálogo pedagógico, la socialización del conocimiento, pero sobre todo intente dar prioridad a la utilización de la investigación como agentes generadores de cambio en el entorno donde ejerce su acción docente.

\section{Metodología}

La presente investigación utiliza los enfoques cuantitativo y cualitativo por considerar que la utilización de un único método o enfoque de investigación puede dar lugar a sesgos metodológicos, en los datos y en los investigadores.

Se realizó un análisis interpretativo del término competencia según la concepción de los directivos del IUTC, donde se aplicó una entrevista.

Se usó la técnica de la encuesta y el análisis interpretativo (hermenéutica). En este caso, la dirección metodológica fue simultánea porque se analizaron los dos instrumentos a la vez, para ver cuáles habían sido los resultados después del análisis estadístico e interpretativo y de esta manera combinar los datos y generar la teoría que iba a fundamentar el perfil de competencias de los docentes de los Institutos de Tecnología de Cabimas.

El cuestionario tipo Likert en su versión preliminar conformado con 85 ítems distribuidos en cuatro (4) dimensiones: 1) competencias personales, 2) competencias docentes, 3) competencias en investigación y 4) competencias de extensión. Esta versión fue sometida a juicio de expertos los cuales evaluaron el instrumento en términos de claridad, tendenciosidad de las respuestas y pertinencia con el indicador.
Luego de realizadas las correcciones sugeridas por los expertos, las cuales fueron en relación a problemas de redacción sin eliminar ningún ítem, el mismo fue sometido a una prueba piloto a un conjunto de 30 sujetos con características similares a la población de estudio. Esto con el propósito de estimar la confiabilidad la cual fue calculada por el método Alpha de Cronbach, dando como resultado 0.9403 , considerado alto.

Con los datos de la prueba piloto, se efectuó la validez del constructo empleando el análisis factorial, el cual como producto del análisis de la matriz rotada permitió obtener la versión definitiva del instrumento, y una nueva tabla de construcción reflejo de una definición acoplada a las condiciones de estudio y definitoria de la manera como se expresa el constructo Competencias Profesionales del Docente Universitario (CPDU) en las condiciones reales. De esta manera se obtuvo la versión definitiva del instrumento, el cual se aplicó a la muestra seleccionada.

\subsection{Análisis estadístico}

El análisis estadístico para el cuestionario se realizó con el SPSS Versión 10. Para obtener los resultados y su posterior análisis, se aplicó la técnica ANOVA, la cual compara las medias de los datos obtenidos de la población encuestada, también se utilizó la prueba de los Múltiples Rangos de Tukey, para ubicar las diferencias significativas de las medias obtenidas, gráficos de medias y la prueba $\mathrm{T}$ para muestras independientes.

La muestra del presente estudio está constituida por 144 docentes ordinarios del IUTC.

\subsection{Análisis e interpretación de los resultados}

Los resultados de la investigación obtenidos por medio del instrumento CPDU fue aplicado con el propósito de elaborar un perfil de competencias profesionales del docente universitario de los Institutos Universitarios de Tecnología. Estos resultados emergieron de las respuestas expresadas por el personal de docentes del IUTC, las cuales revelan el comportamiento de las Competencias Profesionales en el contexto seleccionado.

Con el propósito de comparar las medias alcanzadas por los indicadores, dimensiones y variables, se aplicaron las técnicas estadísticas Anova y Prueba T, para muestras comparadas según la estructura de las dimensiones. Los resultados obtenidos se muestran en gráficos, mientras que los insumos estadísticos de las pruebas aplicadas se incluyen en el Anexo A.

En el gráfico No. 1, se muestran los resultados para la dimensión competencias personales, las cuales revelan diferencias altamente significativas. Asimismo, al aplicar la prueba de múltiples rangos de Tukey se ubicaron dos subconjuntos, correspondiendo el primero de ellos al indicador comunicación, mientras que en el segundo subconjunto se ubicaron los indicadores: sentido del humor, apertura, ética y liderazgo. 


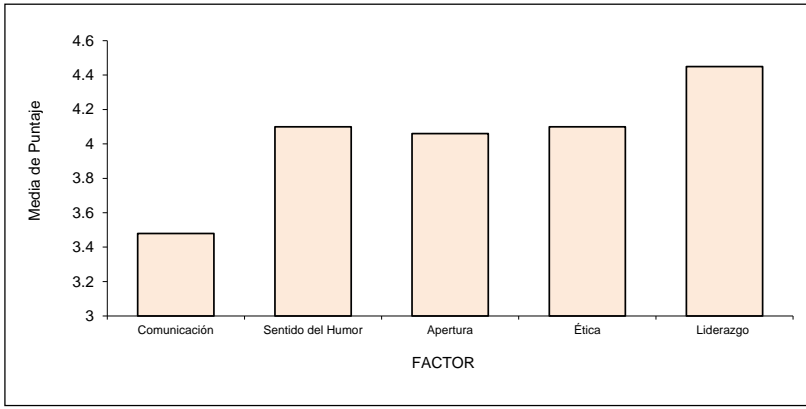

Figura 1: Medias para la dimensión Competencias Personales

Fuente: Gutierrez (2016)

Tal como puede observarse en el gráfico No. 1, el puntaje más alto lo obtuvo el indicador liderazgo (4.4533), seguido de ética (4.1067), apertura (4.0600), sentido del humor (4.1033) y comunicación (3.4844).

En cuanto al liderazgo, los resultados evidencian alta capacidad en los sujetos encuestados para liderar los procesos emprendidos desde la organización. Lo cual confirma el criterio expuesto por Segura (2003), cuando plantea que la competencia del liderazgo se encuentra dirigida a la dirección y orientación del proceso en el aula y fuera de ella, es decir, que incluye dos elementos: uno denominado liderazgo interno y el otro es el liderazgo externo.

El interno se refiere a los valores como docentes, al compromiso con la labor desempeñada y el proceso de planificación académica inherente al desarrollo de los cursos que estén bajo la responsabilidad del mismo; el trabajo desempeñado debe traducirse en acciones concretas, estipulando en forma clara los criterios establecidos para el desempeño y los resultados que se espera de los alumnos.

El liderazgo externo se enfoca en la responsabilidad pública que deben tener los docentes para participar en el desarrollo de proyectos externos y contribuir a mejorar la relación que debe existir en el proceso de interacción entre las actividades del aula y el entorno.

Bajo esta óptica Rugarcia (2001), toma como punto de inflexión la misión del docente, la cual debe promover en el participante la captación de conceptos sobre la profesión y la cultura, a la par del desarrollo de habilidades y el reforzamiento de determinadas actitudes que lo impulsen a ejercer su vocación profesional y humana de cierta manera. Por ello quien ejecute la labor docente debe tener como centro el crecimiento del estudiante, no solo en el tema que se imparte, sino en su desarrollo como individuo.

De igual manera se destaca que la comunicación obtuvo el puntaje más bajo (3.4844) en cuanto al resto de los indicadores; sin embargo, se ubica como suficiente de acuerdo al rango establecido. El resultado revela que en la institución estudiada existen debilidades asociadas al manejo de los intercambios de mensaje y la información entre el personal laboral, lo cual permite afirmar que hace falta implementar mecanismos de comunicación interna para fortalecer los procesos de entendimiento entre los miembros de la organización, traduciéndose como una competencia necesaria en la gestión efectiva del conocimiento dentro de las organizaciones.

Estos resultados no se corresponden con el planteamiento de Cáceres (2003), cuando expresa que el discurso del profesor debe ser presentado con claridad y rigor, la preparación que existe por implicar al alumno en la captación del discurso, la consideración que se presta a las características de la enseñanza realizada en grupo se deben poner en práctica iniciativas especiales para que el discurso captado adquiera raíces en los alumnos.

En el gráfico No. 2, se muestran los resultados para la dimensión competencias docentes, los cuales revelan diferencias altamente significativas. Asimismo, al aplicar la prueba de múltiples rangos de Tukey se ubicaron dos subconjuntos, correspondiendo el primero de ellos al indicador tecnología, mientras que en el segundo subconjunto se ubicaron los indicadores: dominio de la disciplina, formación pedagógica y capacidad de realización.

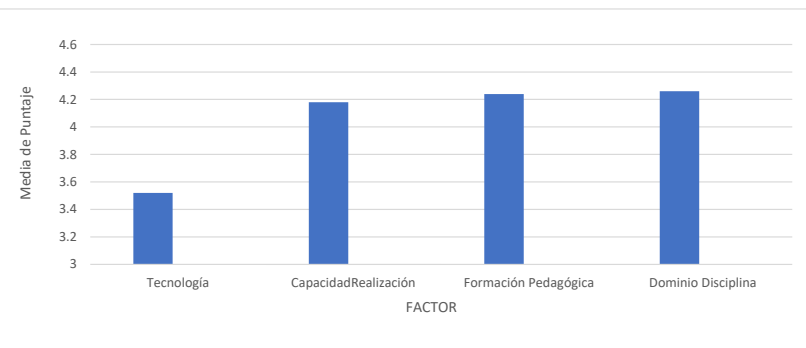

Figura 2: Medias para la dimensión Competencias Personales

Fuente: Gutierrez (2016)

En este sentido, el gráfico No. 2 muestra que el puntaje más alto lo concentró el indicador dominio de la disciplina (4.2667) seguido de formación pedagógica (4.2400), capacidad de realización (4.1867) y tecnología (3.5200).

Sobre la base de estos resultados se destaca que la muestra consultada posee fortalecido el dominio de la disciplina en la que ejerce su acción docente, de lo cual se infiere que los docentes investigados son competentes desde el punto de vista del conocimiento esencial con sus principios y elementos estructurantes, situación que beneficia el desarrollo de procesos educativos y el aprendizaje esperado.

Estos resultados guardan relación con la postura de Tobón (2005), al referir que el dominio de la disciplina como competencia docente caracteriza la facilidad de trasladar de manera asequible y no facilista a los estudiantes los contenidos de las asignaturas que dictan mediante estrategias orientadas en procesos de indagación sistemática, análisis crítico, clasificación, elaboración, reconstrucción y aplicación de la información.

Es un hecho comprobado cuando se tiene dominio de la disciplina, la puesta en acción de un conjunto de herramientas necesarias para procesar la información de manera significativa, acorde con las expectativas individuales de 
los alumnos y a los requerimientos de una situación en particular.

También es preciso resaltar el comportamiento del indicador tecnología, el cual fue designado por los sujetos consultados con el más bajo puntaje (3.5200) en cuanto al resto de los indicadores; sin embargo, se ubica como suficiente de acuerdo al rango establecido. El resultado manifiesta que en la institución estudiada el uso de la tecnología por parte del personal docente no constituye una actividad fundamental; es decir, se evidencia poca valoración hacia el uso y aplicación de la tecnología, más aún al desarrollo tecnológico, lo cual se considera una debilidad extrema por tratarse de una organización cuya misión y visión están orientadas, precisamente, a la tecnología.

En este contexto, el comportamiento demostrado por la tecnología se aleja del planteamiento de Carnoy, Castells, Cohen y Cardoso (1997) cuando sostienen que el gran desarrollo de la ciencia y la tecnología contemporáneas, cuya manifestación más evidente y acelerada es el creciente proceso de informatización de la sociedad, debe reflejarse en el docente universitario en su explotación pertinente a través del uso de las nuevas tecnologías de la información y la comunicación, en función de su labor docente como herramienta insustituible.

Continuando con los resultados se presenta en el gráfico No. 3, los alcanzados por la dimensión competencias en investigación, los cuales no muestran diferencias significativas. Al aplicar la prueba de múltiples rangos de Tukey se ubicaron en un solo subconjunto, donde se aprecia que el puntaje más alto lo obtuvo el indicador producción (3.7467), seguido de desarrollo de investigaciones (3.4667) y aplicación (3.3867).

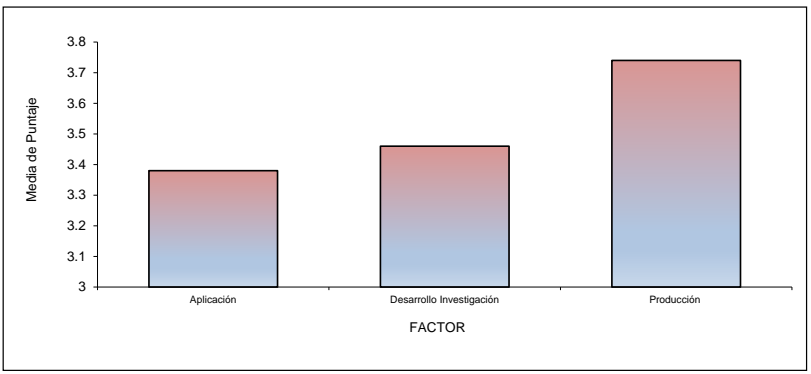

Figura 3: Medias para la dimensión Competencias en Investigación

$$
\text { Fuente: Gutierrez (2016) }
$$

En lo concerniente al comportamiento mostrado por la producción, los resultados permiten asumirla como una competencia fortalecida en los docentes estudiados, revelando que existe dedicación por parte de ellos a generar productos teóricos y conocimientos nuevos con pertinencia a la realidad de la organización, validados científicamente por medio del desarrollo de procesos de investigación.

Estos resultados mostrados, confirman lo expuesto por Padrón (2004) al destacar que la producción en el ámbito de los institutos tecnológicos, sí existe en cuanto al número de trabajos de ascensos y tesis o trabajos de grado para obtener un título, pero la investigación es desarticulada y fragmentada porque los trabajos de investigación no suelen tener nada que ver unos con otros. Si algo debe estar interconectado es precisamente la producción científica. Los trabajos de investigación nacen y mueren dentro de los límites de las circunstancias de un momento y de una persona.

De la misma manera, los resultados muestran para el indicador aplicación el puntaje más bajo (3.3867) en relación al resto de los indicadores; sin embargo, se ubica como suficiente de acuerdo al rango establecido. Estos resultados ubican la aplicación como una competencia en investigación desfavorecida por la muestra consultada, de lo que se infiere la necesidad de promocionar las distintas actividades investigativas que son desarrolladas en la institución y orientar de un modo efectivo su aplicación.

En este orden de ideas, el comportamiento del indicador aplicación no guarda relación con el expresado por Licha (1996) quien considera que la aplicación es una competencia en investigación que requiere de la integración y cohesión en equipos de trabajo. Existe desconexión cuando se aplica alguna investigación, siguiendo un camino divorciado de la docencia y de la extensión. Como se observa se aprecia un esquema individualista para cumplir con algún requisito personal y no institucional.

En el gráfico No. 4 se muestran los resultados para la dimensión competencias de extensión, los cuales fueron calculados por medio de Prueba $\mathrm{T}$ para muestras relacionadas, revelando que no existe diferencia significativa.

Tal como puede observarse en el gráfico No. 4, el puntaje más alto lo obtuvo el indicador interacción social (4.0667), seguido de gerenciamiento de proyectos comunitarios (3.5867).

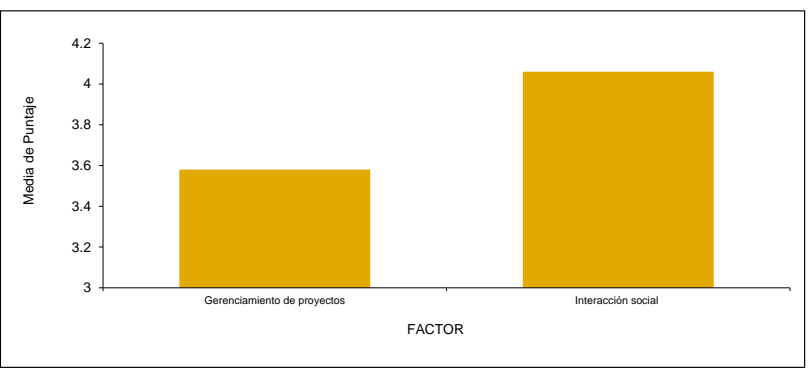

Figura 4: Medias para la dimensión Competencias de Extensión

Fuente: Gutierrez (2016)

De acuerdo con estos resultados, la institución estudiada logra promover de manera competente una interacción social favorable a la vinculación e integración con organismos presentes en el entorno, lo cual permite afirmar que los docentes investigados poseen fortalezas para orientar las competencias de extensión. Estos resultados reafirman el planteamiento de Haverlock y Huberman (1999) referente al aspecto de difusión de las acciones realizadas por el 
docente mediante un proceso de comunicación social con sus compañeros y con su entorno. Por eso, es necesario desarrollar las prácticas investigativas-laborales como manifiesta Lazo (1996), la cual es la forma organizativa de participación activa de los docentes, estudiantes y profesionales al interactuar en la comunidad para ayudar a la solución de problemas que se presenten.

En cuanto al indicador gerenciamiento de proyectos comunitarios, resultó menos favorecido por la muestra encuestada, revelando que hace falta reorientar las actividades gestoras de planes con carácter institucional proyectados hacia la comunidad; es decir, se evidencian debilidades para abordar la realidad comunitaria por medio de proyectos donde la institución pueda gerenciar la integración y vinculación con su entorno.

Estos resultados no se corresponden con el planteamiento de González y González (2001) cuando expresa que la extensión como función universitaria forma parte del proceso educativo, su esencia debe ser la intercomunicación abierta, franca, fresca, horizontal, entre la universidad y todos los sectores de la sociedad. Se trata del despliegue de la acción social de la institución, del ejercicio de su función pública, por medio de una interacción con todos los demás integrantes del cuerpo social para cumplir y asumir su compromiso de participación en el proceso social de creación de cultura y de liberación y transformación radical de la comunidad donde se encuentra inserta.

En el gráfico No. 5 se presentan los resultados para la variable competencias profesionales, los cuales revelan diferencias altamente significativas. Al aplicar la prueba de múltiples rangos de Tukey se ubicaron dos subconjuntos, correspondiendo el primero de ellos a las dimensiones competencias en investigación y competencias de extensión; mientras que en el segundo subconjunto se ubicaron las dimensiones: competencias personales y competencias docentes.

Tal como se muestra en el gráfico No. 5, el puntaje más alto lo obtuvo la dimensión competencias docentes (4.0533) seguido de competencias personales (4.0236), competencias de extensión (3.8267) y competencias de investigación (3.5333).

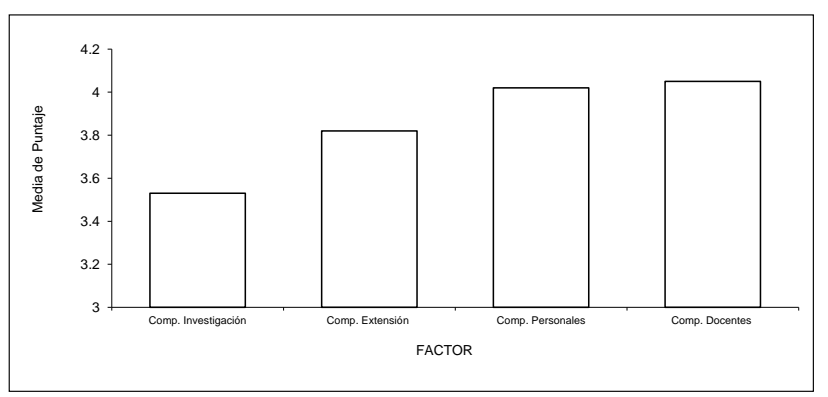

Figura 5: Medias para la Variable Competencias Profesionales

Fuente: Gutierrez (2016)
Estos resultados tienen implicaciones muy importantes para analizar el perfil de competencias profesionales que poseen los docentes de la institución educativa objeto de estudio y en especial, para vislumbrar acciones favorables a la configuración del perfil necesario, de acuerdo a las necesidades más apremiantes de los actores educativos en correspondencia con las demandas sociales presentes en el entorno.

En este sentido, se destaca que las competencias docentes resultaron las más fortalecidas concentrando el puntaje más alto otorgado por la muestra estudiada. Ello revela que los profesores encuestados le dan preeminencia al aspecto docente, se infiere que por ser la gran mayoría profesionales con formación en carreras tecnológicas, hacen mayor hincapié en la necesidad de cumplir a cabalidad con su rol de docente. Para que la institución pueda cumplir sus tareas académicas laborales e investigativas, requiere de profesores preparados, que no solo sepan el contenido específico de su área, sino que sepan enseñar lo que necesita la sociedad, de aquí la necesidad que se enseñe a los profesores a educar, para que los estudiantes aprendan a aprender.

\subsection{Análisis de las entrevistas a directivos}

Tabla 1: Competencias profesionales en la base del diseño curricular

\begin{tabular}{|c|c|}
\hline Concepción: Competencias & Comentario. Directivo 1 \\
\hline $\begin{array}{l}\text { Los cambios en los } \\
\text { modelos educativos } \\
\text { tienen que ir en dos } \\
\text { direcciones: la primera } \\
\text { en tronco común que } \\
\text { consiste en preparar un } \\
\text { ser humano con valores } \\
\text { que lo formen y } \\
\text { fortalezcan su identidad; } \\
\text { la segunda preparar un } \\
\text { profesional con } \\
\text { competencias científicas } \\
\text { y tecnológicas, con } \\
\text { capacidades y } \\
\text { experiencias que le } \\
\text { permitan involucrarse } \\
\text { con el sector productivo } \\
\text { a través de su } \\
\text { preparación en las } \\
\text { Instituciones de } \\
\text { Educación Superior. }\end{array}$ & $\begin{array}{l}\text { Las competencias profesio- } \\
\text { nales son entendidas desde } \\
\text { dos perspectivas: } \\
\text { - Las fundamentan en una } \\
\text { teoría científica de las } \\
\text { capacidades humanas, } \\
\text { para explicar toda } \\
\text { la complejidad de la } \\
\text { formación profesional. } \\
\text { En tal sentido, entiende } \\
\text { las competencias como } \\
\text { capacidades claves } \\
\text { para la realización } \\
\text { de una actividad } \\
\text { profesional y cumplen } \\
\text { un papel de integración } \\
\text { de las capacidades } \\
\text { profesionales. } \\
\text { Esto es muy importante } \\
\text { a considerar, sobre to- } \\
\text { do si se quiere ver a } \\
\text { las competencias como } \\
\text { la base del currículo. }\end{array}$ \\
\hline
\end{tabular}


Tabla 2: Docentes Integrales

\begin{tabular}{|c|c|}
\hline Concepción: Competencias & Comentario. Directivo 2 \\
\hline $\begin{array}{l}\text { Un docente universitario tendría que desarrollar las si- } \\
\text { guientes competencias. } \\
\text { - } \quad \text { Asumir el trabajo docente como una profesión que } \\
\text { requiere de un compromiso personal de tiempo com- } \\
\text { pleto y como un trabajo no lucrativo. } \\
\text { - Debe ser un interesado investigador, en constante } \\
\text { renovación en su área y en el aspecto pedagógico. } \\
\text { - } \quad \text { Caminio de herramientas de comunicación. } \\
\text { - Conocimiento de diálogo inter personal. } \\
\text { - } \quad \text { Tación y liderazgo. } \\
\text { - Abierto a nuevas ideas } \\
\text { - Colaborador }\end{array}$ & $\begin{array}{l}\text { Esta breve reseña presentada ofrece una visión más o } \\
\text { menos amplia de las habilidades, destrezas, actitudes, } \\
\text { aptitudes que los docentes de Educación Superior deben } \\
\text { tener. } \\
\text { Esta es una concepción integral porque considera casi to- } \\
\text { dos los aspectos profesionales desde una posición proac- } \\
\text { tiva acerca de lo que demandan nuestras instituciones } \\
\text { desde una perspectiva crítico social. Vargas (2001). }\end{array}$ \\
\hline
\end{tabular}

Tabla 3: Instaurar condiciones

\begin{tabular}{|l|l|}
\hline \multicolumn{1}{|c|}{ Concepción: Competencias } & \multicolumn{1}{c|}{ Comentario. Directivo 3 } \\
\hline $\begin{array}{l}\text { Es necesario una verdadera preparación profesional, lo } \\
\text { que implica todos los campos, para que los docentes ten- } \\
\text { gan la formación requerida y puedan realizar su trabajo } \\
\text { de la mejor manera posible. }\end{array}$ & $\begin{array}{l}\text { El desarrollo de competencias en los docentes requiere, } \\
\text { según Mertens (2000), la aplicación de los atributos per- } \\
\text { sonales (conocimientos, habilidades, aptitudes y actitu- } \\
\text { Así como en otras profesiones se preparan, el docente } \\
\text { requiere adquirir esas condiciones especiales para hacer } \\
\text { su trabajo. }\end{array}$ \\
$\begin{array}{l}\text { Complemento de esto, que deje de pensarse en esa labor desempeño efectivo. De esta forma la } \\
\text { competencia se constituye en una capacidad real cuando } \\
\text { como un complemento económico salarial, que le resta la } \\
\text { importancia de la actividad y presenta profesionales sin } \\
\text { competencias para cumplir la función de docencia. }\end{array}$ & $\begin{array}{l}\text { lasiones del proceso formativo exigen lograr un } \\
\text { institución. }\end{array}$ \\
\hline
\end{tabular}

Tabla 4: Un desafío para todos

\begin{tabular}{|l|l|}
\hline \multicolumn{1}{|c|}{ Concepción: Competencias } & \multicolumn{1}{c|}{ Comentario. Directivo 4 } \\
\hline $\begin{array}{l}\text { Si se parte de tomar las competencias como el conjunto } \\
\text { de conocimientos y habilidades que todos los sujetos } \\
\text { necesitan para ejercer alguna actividad, en este caso la } \\
\text { docencia, se requiere pensar no solo en las capacidades y } \\
\text { habilidades disciplinarias, sino también las referidas al } \\
\text { comportamiento social, profesional, a las actitudes, a la } \\
\text { capacidad de innovar y a las capacidades éticas. }\end{array}$ & $\begin{array}{l}\text { Se hace necesario impone } \\
\text { Vetencias en los docentes universitarios comprende tres } \\
\text { aspectos: }\end{array}$ \\
& $\begin{array}{l}\text { L La potencialidad para aprender a realizar el trabajo } \\
\text { - La disposición o motivación para realizarlo. }\end{array}$ \\
& $\begin{array}{l}\text { La necesidad de entender la complejidad de la com- } \\
\text { petencia en el orden estructural en el que se incluyen } \\
\text { componentes no solo cognitivos, sino también personales } \\
\text { y motivacionales, es también destacada por Spencer- } \\
\text { Spencer (1996). }\end{array}$ \\
\hline
\end{tabular}


Tabla 5: Metodología de la Enseñanza

\begin{tabular}{|l|l|}
\hline \multicolumn{1}{|c|}{ Concepción: Competencias } & \multicolumn{1}{c|}{ Comentario. Directivo 5 } \\
\hline Los docentes universitarios están comprometidos con la for- & Se observa una gran preocupación por el aspecto netamente de \\
mación de personas que desarrollan competencias laborales & aula, obviando otros elementos de importancia. La concepción \\
necesarias para competir, es por ello, que los ambientes de & sobre el desarrollo de competencias es muy limitado y Gonz- \\
aprendizaje deben cambiar de modelos tradicionales a modelos & ci (1996), expresa que la competencia se concibe como una \\
de participación de los alumnos dentro del aula. Las estrategias & compleja estructura de atributos necesarios para el desempeño \\
empleadas deben ser más dinámicas y con total organización & de situaciones específicas. Es una compleja combinación de \\
de la clase en trabajos cooperativos que propicien el trabajo en & atributos, valores y habilidades y las tareas que se tienen que \\
equipo. & desempeñar en determinadas situaciones. \\
\hline
\end{tabular}

Tabla 6: Quehacer Profesional, Ética y Gerencia

\begin{tabular}{|c|c|}
\hline Concepción: Competencias & Comentario. Directivo 6 \\
\hline $\begin{array}{l}\text { En la actualidad existen muchas expectativas e iniciativas en } \\
\text { cuáles deben ser las competencias que identifican al profesor } \\
\text { universitario; sin embargo, también hay consenso en cuanto } \\
\text { a la importancia de la generación de conocimiento en las } \\
\text { instituciones actuales. De igual manera, pienso que será difícil } \\
\text { convencer a los profesores profesionales de otras áreas (inge- } \\
\text { nieros, economistas) que durante años han dictado sus clases } \\
\text { sin ninguna preparación pedagógica, siendo estas competencias } \\
\text { fundamentales en las actividades de aula ¿Qué se puede hacer } \\
\text { con esos profesores? Además, se debe combatir el ingreso de } \\
\text { nuevos docentes con criterios distintos a los académicos, es } \\
\text { decir, que por ser amigo de las autoridades directivas arreglan } \\
\text { su ingreso para beneficiar a la persona y no a la institución. }\end{array}$ & $\begin{array}{l}\text { Estas expresiones nos llevan a la reflexión que ética, quehacer } \\
\text { profesional y gerencia, se encuentran íntimamente relaciona- } \\
\text { dos. Es muy importante manejarse con una conducta ética } \\
\text { mediante el ejercicio de prácticas congruentes con los princi- } \\
\text { pios institucionales. Una de las funciones con mayor exigencia, } \\
\text { por su alto compromiso con las nuevas generaciones y con } \\
\text { los destinos de un país, es la del educador. Bajo esta óptica, } \\
\text { Rugarcia (2001), expresa que la misión del docente es la de } \\
\text { promover en el alumno la adaptación de conceptos sobre la } \\
\text { profesión y la cultura, a la par el desarrollo de habilidades y } \\
\text { actitudes que lo impulsen a ejercer su vocación profesional y } \\
\text { humana de cierta manera. Según González (1981), el profesor } \\
\text { universitario, cualquiera que sea su especialidad, ingeniería, } \\
\text { derecho, medicina, es solicitado por la institución no como } \\
\text { profesional técnico, sino como educador. }\end{array}$ \\
\hline
\end{tabular}

Tabla 7: Quehacer Profesional, Ética y Gerencia

\begin{tabular}{|c|c|}
\hline ción: Co & 7 \\
\hline $\begin{array}{l}\text { En la gran mayoría de los sistemas educativos en el mundo } \\
\text { no existen instituciones creadas para formar los docentes uni- } \\
\text { versitarios en el instituto como son carreras tecnológicas se } \\
\text { llega a cumplir ese rol y cuando está en el campo, asume el } \\
\text { papel de enseñante transmitiendo aquella información y saber } \\
\text { comunicarlo de la mejor forma intuitiva porque la mayoría } \\
\text { desconoce las herramientas didácticas, enfoques pedagógicos } \\
\text { que son útiles para el ejercicio docente. Este modelo didáctico } \\
\text { centrado en la transmisión de la información, muchas veces mal } \\
\text { llamado conocimiento, ha sido lo prevaleciente para enseñar en } \\
\text { estas instituciones. } \\
\text { Aquí surge el nuevo perfil y las llamadas nuevas competencias } \\
\text { del docente universitario. Al respecto opino que debería concep- } \\
\text { tualizar lo que se considerará competencias profesionales del } \\
\text { docente universitario. Sin embargo, considero que el modelo } \\
\text { debe tomar en cuenta: el saber ser, competencias personales, } \\
\text { desde el sabe aprender, competencias académicas desde el } \\
\text { saber hacer, docencia competencias pedagó gicas, sumando las }\end{array}$ & $\begin{array}{l}\text { La competencia profesional se manifiesta en la } \\
\text { tanto es en la actuación profesional que se expre } \\
\text { cimientos, hábitos, habilidades, motivos, sentimie } \\
\text { que de forma integrada regulan la actuación del } \\
\text { búsqueda de soluciones a los problemas profesion } \\
\text { La actuación profesional del docente se manif } \\
\text { planos: el interno que se refiere a la reflexión y las } \\
\text { externo es conductual. Según González ( } 2002) \text {, la } \\
\text { profesional en sus niveles superiores de desarrollo } \\
\text { monía y la integridad del sentir, el pensar, el hacer } \\
\text { su actuación profesional. Se quiere decir que un p }\end{array}$ \\
\hline
\end{tabular}


Tabla 8: Competencias Pedagógicas del Docente Universitario

\begin{tabular}{|c|c|}
\hline Concepción: Competencias & Comentario. Directivo 8 \\
\hline $\begin{array}{l}\text { Desarrollar, redefinir y fortalecer las competencias peda- } \\
\text { gógicas del docente universitario es aportar en su cua- } \\
\text { lificación e incidir en la formación integral de los estu- } \\
\text { diantes. Para optimizar la formación docente se requiere } \\
\text { la implementación de un proceso pedagógico integral. El } \\
\text { docente es el eje fundamental en el proceso educativo } \\
\text { dentro del mejoramiento, la calidad y la excelencia. Es } \\
\text { así, como desde el concepto de competencia pedagógica } \\
\text { como aquel complejo conjunto de capacidades, conoci- } \\
\text { mientos, habilidades y valores demostrables por el do- } \\
\text { cente entre el saber-hacer, el cómo hacer y el saber ser } \\
\text { pedagógico de manera que responda al proceso integral } \\
\text { de formación humana entre él y los actores implicados en } \\
\text { el proceso educativo }\end{array}$ & $\begin{array}{l}\text { En este comentario se observa que existe una concepción } \\
\text { restringida sobre las competencias y conciben al docente } \\
\text { como el eje fundamental. El dominio pedagógico es } \\
\text { importante pero no es lo único, es necesaria la integra- } \\
\text { lidad. No obstante, al respecto Zurita, citado por Silva } \\
\text { (1993), llama la atención acerca de los estilos docentes } \\
\text { dominantes en este nivel educativo, en el cual según él } \\
\text { prevalecen relaciones de dominación y control y muy } \\
\text { escasamente relación de comunicación creadora, por lo } \\
\text { que resalta el error que se comete, al no intentar vencer } \\
\text { esta tendencia descrita por el autor como pedagogismo } \\
\text { castrante, autoritario, regido por los principios de depen- } \\
\text { dencia. }\end{array}$ \\
\hline
\end{tabular}

Tabla 9: Predominio de la Tecnología

\begin{tabular}{|l|l|}
\hline \multicolumn{1}{|c|}{ Concepción: Competencias } & \multicolumn{1}{c|}{ Comentario. Directivo 9 } \\
\hline La actual sociedad del conocimiento o más globalizada, & No necesariamente el que estemos en el siglo XXI signifi- \\
exige a los formadores nuevas competencias, comenzan- & ca que los docentes universitarios seamos unos profesio- \\
do por el término de competencias para enseñar, empleo & nales con grandes conocimientos en tecnología. Ese siglo \\
de nuevas tecnologías de información y comunicación, & exige un nuevo tipo de docente, con nuevos conocimien- \\
realizar su trabajo con la mejor calidad, donde el predo- & tos y responsabilidades, que investigue, que se actualice \\
minio, sea lo tecnológico sobre los aspectos humanos, el & constantemente, que fomente en los estudiantes valores \\
producir sobre el sentir, el maquinismo sobre la organiza & no solo tecnológicos sino para la vida en comunidad. \\
ción. El docente universitario debe formar para el trabajo & Puig (1996) expresa que la realidad económica, social, \\
productivo, empleando tecnologías de última generación, & política y cultural del país donde vive, los conceptos que \\
pero sin olvidarse que es formar personas, que tomen y & predominan a nivel productivos, serán los que indiquen la \\
actúen las mejores decisiones, pensando en los demás y & clase de formación y el modelo pedagógico del profesor \\
no solo en el éxito o el confort material. & universitario. \\
\hline
\end{tabular}

Tabla 10: Responsabilidad Social del Docente

\begin{tabular}{|l|l|}
\hline \multicolumn{1}{|c|}{ Concepción: Competencias } & \multicolumn{1}{c|}{ Comentario. Directivo 10 } \\
\hline Se hace pertinente introducir la reflexión en torno de & Los docentes deben definir el grado de responsabilidad \\
una competencia fundamental para el ejercicio docente & social que este rol implica por lo menos en nuestro \\
y es justamente la de la capacidad reflexiva, crítica y & contexto. Creo que en definitiva aquí radica la esencia \\
constructiva que debe poseer el mismo. Muchas veces & docente del siglo XXI y no se trata de promover ideas \\
reflexiono sobre qué es lo que en realidad necesitan los & de izquierda o de derecha o colores políticos. Galdona \\
estudiantes de sus docentes, porque el conocimiento está & $(2000)$ afirma que es necesario generar procesos de re- \\
al alcance de todos, no lo sabemos todos y si existe un & flexión en donde el estudiante se pregunte y se busque \\
estudiante brillante, que los hay, posiblemente nos pueda & sus propias respuestas en torno a una realidad cambiante \\
enseñar. & como la actual. \\
\hline
\end{tabular}


Tabla 11: Competencias profesionales en la base del diseño curricular

\begin{tabular}{|c|c|}
\hline Concepción: Competencias & Comentario. Directivo 11 \\
\hline $\begin{array}{l}\text { Personalmente considero } \\
\text { que debe haber una } \\
\text { redefinición del perfil del } \\
\text { docente universitario que } \\
\text { implica ser: } \\
\text { - Un promotor de enfo- } \\
\text { ques trascendentes } \\
\text { - Un productor de alian- } \\
\text { zas con proyectos polí- } \\
\text { ticos organizacionales } \\
\text { Un manejador de arti- } \\
\text { culaciones en los nue- } \\
\text { vos circuitos de infor- } \\
\text { mación } \\
\text { Un especialista } \\
\text { en contenido y } \\
\text { comunicación } \\
\text { Un mediador entre el } \\
\text { conocimiento, la infor- } \\
\text { mación y los estudian- } \\
\text { tes } \\
\text { Un promotor y facilita- } \\
\text { dor de la creatividad y } \\
\text { el pensamiento lógico- } \\
\text { crítico }\end{array}$ & $\begin{array}{l}\text { La gestión del conocimiento, en } \\
\text { la actual sociedad, exige la re- } \\
\text { organización interna de los pro- } \\
\text { cesos que ocurren en las Ins- } \\
\text { tituciones de Educación Supe- } \\
\text { rior y por tanto la mejora de } \\
\text { la docencia y de la investiga- } \\
\text { ción para facilitar el desarrollo } \\
\text { de una institución competitiva, } \\
\text { adaptada a las nuevas deman- } \\
\text { das de la sociedad. En efecto, } \\
\text { se hace necesario el replantea- } \\
\text { miento del perfil del docente en } \\
\text { un modelo de aprendizaje co- } \\
\text { laborativo, donde se conceptúe } \\
\text { como un líder en gestión de la } \\
\text { información, lo cual requerirá } \\
\text { del dominio de conocimientos, } \\
\text { habilidades, destrezas y actitu- } \\
\text { des necesarios para abordar el } \\
\text { cambio. } \\
\text { Pero también es importante co- } \\
\text { mo expresa Salinas, las nece- } \\
\text { sidades de un docente univer- } \\
\text { sitario básicamente tienen dos } \\
\text { facetas: cuestiones relacionadas } \\
\text { con el conocimiento y con los } \\
\text { procesos de comunicación y di- } \\
\text { dácticos, la investigación y el } \\
\text { trabajo comunitario. }\end{array}$ \\
\hline
\end{tabular}

\subsection{Perspectivas teóricas. Fundamentación o base para un enfoque integral de las competencias profesionales del docente universitario}

La educación superior se enfrenta a una serie de retos en el mundo que se transforma, por ello debe revisar su misión y redefinir muchas de sus tareas sustantivas, en especial aquellas que se relacionen con las necesidades de la sociedad en materia de aprendizaje y superación continua. Un elemento de gran trascendencia en su misión se encuentra dirigido a destacar las tareas de las Instituciones Universitarias de Tecnología.

La mejora de la calidad del proceso enseñanza aprendizaje pasa necesariamente por la transformación del pensamiento y de los sentimientos de los docentes. Para ello la educación superior necesita de la calidad del personal docente, de los programas, estudiantes y de la infraestructura. Por ende, también es importante y necesario que para que las instituciones puedan cumplir a cabalidad en sus funciones, deberían tomarse medidas adecuadas en materia de investigación, programas de entrenamiento adecuados para la formación de personal que estimulen la innovación permanente y que aseguren condiciones profesionales apropiadas a los docentes a fin de garantizar la excelencia.

La idea de la educación durante toda la vida, de la educación permanente es aplicable al profesional de la educación superior, pues el profesor universitario en atención a la tarea que desarrolla requiere que disponga de posibilidades para su formación continua.

La posición que asume la investigación en cuanto a cómo debe ser la formación docente, es aquella que se concibe como el proceso permanente de adquisición, estructuración y reestructuración de conocimientos, habilidades y valores para el desempeño de la función docente. De tal manera, que la formación docente debe ser continúa y llevarse a cabo toda la práctica docente tomándose dicha práctica como eje formativo estructurante. La investigación fue aplicada en el Instituto Universitario de Cabimas - Venezuela.

La educación permanente puede definirse como el proceso que mejora los conocimientos referentes a la actuación, estrategias y las actitudes de quienes trabajan en las Instituciones de Educación Superior. La finalidad prioritaria de la formación permanente es mantener actualizado al docente en los aspectos relacionados con el que hacer docente. Con base a los resultados obtenidos en la investigación mediante el análisis de los datos, tanto los docentes como los directivos abogan o enmarcan el perfil de competencias en una concepción integral y humanista. De ahí se explicita que el concepto asumido sobre las competencias profesionales del docente universitario, es el siguiente conjunto de cualidades fundamentales en el orden científico, pedagógico valorativo, cualidades enfocadas a las direcciones básicas que debe asumir el docente en su área específica de trabajo. Pero para que estas características esenciales se potencien y desplieguen es necesario que el papel que tradicionalmente han desempeñado, los dos procesos de enseñanza-aprendizaje se transforme en forma efectiva.

El papel del docente y del estudiante deben ser vistos de modo integral ya que, ambos constituyen una unidad, en ese proceso lo tradicional y lo emocional, lo cognitivo y lo afectivo, se deben complementar, la búsqueda de la verdad y la formación de valores deben ser una unidad insuperable. Estos son aspectos inherentes al proceso académico docente que lo hacen aún más complejo.

Una de las implicaciones que se deriva de esta visión integral para quienes desean el desarrollo de la institución y para el propio docente, es comprender que la realidad donde se realiza el profesor no es estática, pero tampoco inestable; no es fragmentada pero tampoco homogénea o indiferenciada, eso sí, es muy compleja.

En una Institución de Educación Superior como el Instituto Universitario de Tecnología de Cabimas - Venezuela, donde el mayor porcentaje de sus profesores son ingenieros, los cuales no poseen formación pedagógica en su área de pregrado, la mayoría ejerce la función de docentes guiados por el empirismo, por el ejemplo de profesores que tuvieron en su vida como estudiante, eso justifica la necesidad del conocimiento en investigación y capacitación pedagógica. Cuando se plantea la necesidad de crear un modelo de profesor universitario, se busca con ello crear un perfil que sirva de base o guía a las necesidades de formación y ca- 
pacitación integrándose la actividad docente, investigativa y extensionista como una de las vías principales para la superación de la calidad del profesor. Lo anterior no se disocia de la formación humanista de los profesionales.

Formar un profesional competente, responsable y comprometido con el desarrollo social, significa que posea los conocimientos y habilidades que le permitan desempeñarse con éxito en la profesión y sustituirlo por una concepción más amplia y humana del profesional, entendido como un sujeto que orienta su actuación con independencia y creatividad sobre la base de una sólida motivación profesional que le permita perseverar en la búsqueda de soluciones a los problemas profesionales auxiliado por sus conocimientos y habilidades en una óptica creativa.

Luego de realizar la fundamentación para un enfoque integral de competencias, se estableció el perfil. Este se realizó tomando en cuenta los resultados de los dos instrumentos y se tomaron en cuenta los siguientes aspectos que definen atributos y rasgos de conducta deseables en el docente universitario.

Perfil profesional referido al:

- Ser, a los valores, la personalidad

- Saber teórico intelectivo, del ejercicio de su profesión o área de formación en el pregrado

- Saber hacer con expresión en el comportamiento psicomotriz y destrezas de ejecución

\subsection{Perfil del docente}

El perfil del docente es una definición de los rasgos, atributos, cualidades y capacidades que configuran su profesionalidad; se define en términos de Saber, Saber Hacer y del Ser que serán el resultado del proceso de formación inicial y contínua del educador.

Este conjunto de atributos en general, se organiza en áreas:

a. Personal: que describe atributos y rasgos referidos al educador como persona integral, que dotado de potencialidades y cualidades, es un miembro importante de la familia y la comunidad en que el corresponde vivir.

b. Académica: en estas áreas se integran el conjunto de saberes referidos a las disciplinas que sustentan el vasto campo de la educación, la pedagogía y los fenómenos naturales y sociales provenientes de la cultura sistematizada.

c. Pedagógico: en esta área se incluyen los atributos o rasgos que se refieren al ejercicio teórico metodológico del quehacer de los procesos de enseñanza y aprendizaje. Implica todo lo referido al quehacer docente en el aula, la institución y la comunidad; se refiere al desempeño del trabajo profesional como parte del sistema educativo.

Se trata del establecimiento de un conjunto articulado de competencias académica-profesionales que se complementan para hacer posible una práctica pedagógica de mayor calidad.
La definición de perfiles es una tarea compleja; recientemente se ha incorporado al trabajo pedagógico-curricular el enfoque por competencias. La misma, según uno de sus autores Le Bortef, la define como: Un "saber actuar" basado en la construcción personal que va a depender de los saberes que se poseen, las experiencias, la personalidad y los recursos del entorno de los cuales se pueden echar mano; así, el actuar con competencia va a estar condicionado por los "recursos incorporados" y los "recursos del entorno", conocimiento combinatorio. Las competencias no se aprenden como totalidad acabada; se aprenden recursos para "actuar con competencias".

Lo que se refiere a:

a. Saber conceptual: referido al conocimiento de ideas, datos, hechos, principios, leyes.

b. Saber procedimental: habilidades y destrezas

c. Saber actitudinal: referido a hábitos, valores, creencias, actitudes, ideales, entre otros.

Las competencias se reconocen como una secuencia y no como operaciones aisladas y están fuertemente vinculadas a la práctica.

Todo lo anterior tiene implicaciones para las Instituciones de Educación Superior; un perfil basado en competencias no es una declaración verbal de intenciones; se trata por el contrario de un instrumento de trabajo para guiar, orientar, dar unidad y coherencia al trabajo de formación inicial y continua del docente.

Lo anterior será aplicado en las Instituciones de Educación Superior de la siguiente manera:

a. El docente deberá transformar sus paradigmas formativos de expositor a facilitador donde la clase ya no será una audiencia que escucha sino un laboratorio viviente, un taller donde se movilizan recursos y se producen respuestas.

b. La formación en competencias implica redimensionar aspectos sustantivos de los planes y programas de estudio.

Los problemas de la formación continua podrían sintetizarse, ya más cerca del campo pedagógico, en el conjunto de los análisis del aprendizaje desde la perspectiva del constructivismo y, en especial, sobre la naturaleza de los contenidos. Se considera que este enfoque es útil para propiciar el aprendizaje de las competencias del perfil propuesto. Tal como se ha indicado este trabajo pretende antes que ofrecer un listado de los rasgos o atributos, ofrecer una serie de ideas y elementos para el análisis sobre los siguientes aspectos:

\subsection{Propuesta para el perfil de competencias del docente universitario}

\subsection{1 Área Personal}

1) Tener vocación docente

2) Poseer salud física y mental; y una personalidad equilibrada

3) Mostrar actitud permanente hacia el cambio 
4) Tener espíritu de conciliación

5) Demostrar habilidad para la comunicación permanente y eficaz

6) Poseer sensibilidad social hacia el mejoramiento de la calidad de vida de su comunidad

7) Demostrar habilidad de liderazgo participativo y comunitario

8) Evidenciar una actitud hacia el mejoramiento profesional continuo

9) Ser promotor de actividades recreativas, culturales, deportivas y de protección al medio ambiente

10) Capaz de aprender a ser mediante la tolerancia, la solidaridad y la reflexión

11) Mantener un alto nivel de auto estima que le permita demostrar seguridad en sí mismo

12) Mantener una actitud de investigación permanente

13) Mantener apariencia física y lenguaje adecuado

\subsection{2 Área Social}

1) Asumir niveles de compromiso en la solución de problemas comunales, regionales, nacionales e internacionales, que permitan una mejor calidad de vida.

2) Ser capaz de ofrecer los valores de la familia como base fundamental para el desarrollo de la sociedad.

3) Propiciar acciones conducentes al logro de la paz.

4) Practicar los principios democráticos y de respeto a los derechos de los demás.

5) Ser modelo de acciones tendientes a propiciar una cultura de paz.

6) Poseer sensibilidad social.

7) Propiciar el uso racional y sostenible de los recursos y la conservación del medio ambiente.

8) Ser capaz de utilizar los conocimientos científicos y tecnológicos en beneficio de su desarrollo personalsocial que le permita comprender y aportar a las grandes transformaciones del mundo que le rodea

9) Incentivar la conservación de la salud individual y colectiva.

10) Propiciar el trabajo cooperativo y la solidaridad humana.

\subsection{3 Área Profesional}

1) Estimular la autonomía de los alumnos y apoyarlos según las necesidades específicas de aprendizaje, promoviendo a su vez el trabajo colaborativo.

2) Propiciar un ambiente que genere confianza, para el desarrollo de la autoestima y el aprendizaje permanente.

3) Desarrollar una labor pedagógica a través de un proceso de investigación científica de la realidad social y educativa, enfatizando en la capacidad de búsqueda personal de información actualizada.

4) Obtener conocimientos pedagógicos y didácticos de las teorías educativas actuales y de las nuevas tendencias.

5) Poseer una sólida formación en el campo científico, humanístico y tecnológico.
6) Desarrollar un espíritu de permanente formación profesional ante los cambios o innovaciones educativas, científicas y tecnológicas.

7) Lograr la concienciación de la necesidad de mantenerse en un proceso constante de autoevaluación y heteroevaluación de su desarrollo personal y profesional.

8) Promover el trabajo colaborativo y de apoyo docentedocentes en función del intercambio de experiencias, enriquecimiento profesional y mejoramiento de la calidad de la labor educativa.

9) Visualizar la responsabilidad del docente ante el desarrollo de sus competencias profesionales para proyectarlas a sus alumnos en su labor educativa.

10) Desarrollar la capacidad de actuar como facilitador, seleccionando y utilizando métodos y recursos apropiados para el logro de aprendizaje significativo.

11) Retomar una mística de trabajo basada en la vocación docente de compromiso y entrega ante su labor educativa.

12) Desarrollar la teoría curricular integrada a una realidad y al contexto social inmediato por medio de adecuaciones.

13) Desarrollar la creatividad, autoaprendizaje, transferencia de conocimientos, habilidades, destrezas y actitudes en sí mismo y en sus discentes.

14) Ser capaz de orientar el proceso de aprender a aprender, aprender a ser, aprender a hacer y aprender a convivir en la aplicación de la didáctica como elemento fundamental de aprendizaje.

15) Aplicar la tecnología de avanzada como herramienta en el desempeño de su labor docente.

16) Conocer sus derechos y deberes establecidos en las leyes para el ejercicio de su profesión.

\section{7 Área Técnico-Pedagógica}

1) Planificar, organizar, coordinar, evaluar y conducir el proceso educativo del aula y la institución con base a una amplia perspectiva de la realidad local, nacional e internacional.

2) Considerar las necesidades y diferencias individuales de los alumnos, así como sus intereses y expectativas al programar y desarrollar actividades y tareas pedagógicas.

3) Incorporar en su práctica cotidiana procedimientos metodológicos y evaluativos acordes con los nuevos modelos pedagógicos.

4) Organizar y conducir el proceso de enseñanza de manera efectiva mediante el empleo de estrategias didácticas que aseguren la actividad participativa e innovadora de los alumnos.

5) Coordinar la introducción de innovaciones pedagógicas en el desarrollo de las actividades y tareas educativas, acorde a los adelantos científicos, humanísticos y tecnológicos.

6) Investigar, interpretar y comprender los procesos de enseñanza-aprendizaje de manera tal que seleccione y 
utilice las estrategias, técnicas y recursos más adecuados a las necesidades e intereses de aprendizaje de sus estudiantes.

7) Desarrollar una variedad de situaciones de aprendizaje y estrategias metodológicas que respondan a las características del alumno y su contexto socio-cultural.

8) Razonar, analizar, discernir y resolver las situaciones en el aula, a fin de liderizar y orientar los procesos de conducta de sus alumnos.

9) Considerar los intereses y expectativas de la comunidad educativa.

10) Poseer una amplia preparación pedagógica que asegure la calidad en su desempeño docente.

11) Demostrar un criterio de competencia global articulando nuestra realidad técnico-docente con los avances tecnológicos, innovadores sin dejar de enfocar la problemática social.

12) En cuanto a la dimensión técnico-pedagógica, se sugieren cuatro categorías de competencias:

a. Habilidades para planificar, administrar de manera creativa.

b. Habilidades para aplicar técnicas y estrategias metodológicas apropiadas a los cambios actuales

\section{Conclusiones}

Las competencias docentes obtuvieron el puntaje más alto, seguido de las competencias personales, las de extensión y, por último, las competencias de investigación. Esto revela lo siguiente:

Los profesores le dan preeminencia al aspecto docente, se concluye que eso es motivado a que la gran mayoría de los docentes son egresados de carreras tecnológicas y hacen mayor hincapié en la necesidad de cumplir cabalmente con el rol docente. En consideración con las competencias personales, la actitud individual personal del docente son determinantes porque el ser humano tiene talentos y virtudes que se desarrollan cuando encuentran un clima propicio, es de allí la importancia del docente para crear ese ambiente favorable que ayudará al estudiante a enriquecer de conocimientos y experiencias y así lograr una transformación eficaz en su aprendizaje. En relación a las competencias de extensión, resultan disminuidas. El docente debe internalizar que a través de la extensión se justifica la pertinencia y compromiso social de la universidad con la comunidad. La acción comunitaria permite la detección de potencialidades existentes que posee la comunidad y que pueden ser aprovechadas por los docentes para la contextualización y desarrollo del proceso pedagógico de forma tal que prepare al estudiante para la vida.

Con relación a las competencias en investigación son las que arrojaron el puntaje más bajo. Esto traduce que el docente debe revisar la práctica universitaria cotidiana para reflexionar sobre el impacto que genera el dominio de procesos de investigación y sistematizar acciones que se conviertan en puntos de partida para aumentar la producción científica y tecnológica que debe destacar a un docente universitario. La entrevista aplicada a los directivos, abogan por un perfil de competencias integral dentro de una perspectiva humanística, dando énfasis a los criterios pedagógicos.

\section{Referencias Bibliográficas}

Barrón, C. (2000). La formación en competencias y certificación profesional. México: Editorial Valle.

Carnoy, M., Castells, M., Cohen, S., y Cardozo, F. (1997). La información en la nueva economía global. reflexiones en un mundo cambiante. Pennsylvania: The Pennsylvania State University.

Cáceres, M. (2003). La formación de los profesores universitarios: Una propuesta en el proceso de profesionalización del docente. Cuba.

Cortina, A. (2001). El mundo de los valores. Ética mínima y educación. Santa Fe de Bogotá: Editorial El Buho.

González, M. (2002). La orientación profesional en la educación superior. una alternativa teoricametodológica para la formación de profesionales competentes. La Habana. Cuba. (Ponencia III Convención Internacional de Educación Superior)

Ortiz. (2002). Las competencias profesionales del ingeniero mecánico. una alternativa de diseño curricular. Universidad de Puerto Rico.

Padrón-Guillen, J. (2004). Los 7 pecados capitales de la investigación universitaria tercermundista. Informe de investigaciones educativas, VOL. XVIII, 69-80.

Rugarcia. (2001). Hacia el mejoramiento de la educación.

Tobón, S. (2005). Formación basada en competencias. pensamiento complejo, diseño curricular y didáctica. Bogotá: Eco.

Recibido: 25 de noviembre de 2018

Aceptado: 15 de marzo de 2019 